\title{
Glioblastoma cells negative for the anti-CD133 antibody AC133 express a truncated variant of the CD133 protein
}

\author{
TARYN L. OSMOND, KATE W.R. BROADLEY and MELANIE J. McCONNELL \\ Malaghan Institute of Medical Research, Wellington 6012, New Zealand \\ Received February 19, 2010; Accepted March 22, 2010
}

DOI: 10.3892/ijmm_00000418

\begin{abstract}
The transmembrane glycoprotein CD133 is a marker commonly used for isolation and analysis of putative cancer stem-like cells. However, analysis of CD133 expression is potentially confounded by the fact that two of the commonly used anti-CD133 antibodies, AC133 and 293C, only recognize CD133 that has undergone glycosylation. Therefore, our aim was to thoroughly examine antibody recognition and mRNA expression of CD133 in glioblastoma multiforme. Glioblastoma cell lines and primary cultures obtained from resected tumor tissue were analyzed by real-time PCR, Western blot analysis, and flow cytometry for CD133, and immunofluorescence was used to determine cellular localization. The AC133 and 293C antibodies did not detect any CD133 on the surface of the glioblastoma cells despite the fact that a protein was detected using C24B9, an anti-CD133 antibody that recognizes an unglycosylated epitope. This CD133 variant was truncated $(\sim 16 \mathrm{kDa})$ and, unlike typical expression of full-length CD133 protein, was found throughout the cytoplasm instead of localized to the plasma membrane. Levels of mRNA and protein for the variant increased with stress, indicating potential for it to be a functional molecule. Because AC133 and 293C antibodies do not detect all CD133 variants in glioblastoma cells, alternate detection methods need to be utilized for complete analysis of CD133 expression and for accurately determining the relationship between CD133 and cancer stem-like cells.
\end{abstract}

\section{Introduction}

CD133, also known as prominin-1, is an 858 amino acid long, $\sim 115 \mathrm{kDa}$ plasma membrane glycoprotein (1) with five transmembrane domains (1-9). It contains two short cytoplasmic loops and two large glycosylated extracellular loops $(1,9)$, with an extracellular $\mathrm{N}$-terminus and cytoplasmic C-terminus $(1,10)$. The glycosylated extracellular domains are 255 and 290 amino acids long with $8 \mathrm{~N}$-linked glycosylation

Correspondence to: Dr Melanie J. McConnell, Malaghan Institute of Medical Research, Entrance 7, Kelburn Parade, Wellington 6012, New Zealand

E-mail: mmcconnell@malaghan.org.nz

Key words: CD133, glioblastoma, stress, truncation sites in total (10). Five alternative promoters allow transcription of various alternate mRNA isoforms $(4,9)$. The function of CD133 is unknown $(4,5)$, however it has been described as a cell surface marker of stem cells $(4,5,11)$, including the stemlike cancer stem cells $(5,7,9,12)$, and is found on different types of stem and cancer cells (4). Alternate mRNA isoforms may have alternative functions, and regulation of translation and post-translational modifications may also impact the function of CD133 in cells.

CD133 was originally discovered in human hematopoietic stem cells $(2,10)$ and is also found in normal colon epithelial cells $(5,9)$. Many previous studies $(7,8,12,13)$ have identified the presence of CD133 on stem-like cells from glioblastoma (GBM) as well as many other cancers including liver, prostate and colon. These 'cancer stem cells' are a subset of tumor cells that can self-renew $(6,9,14)$ due to oncogenic mutations (15); can differentiate into multiple lineages $(9,13,14)$; and are responsible for growth of primary tumors $(4,14,16)$, tumor maintenance $(13)$, and re-growth of metastatic tumors (16). Cancer stem cells are resistant to radiation and chemotherapy $(8,9,13)$ and can remain and cause a tumor to re-grow even after $99 \%$ of the tumor has been eradicated $(3,9,16)$.

While there is a correlation between CD133 positivity and cancer stem-like cells, there is also evidence that CD133 negative cells have stem-like properties $(4,6)$. CD133 positive cells have been shown to initiate tumors and be associated with metastasis and cancer progression (3), however CD133 negative cells have also been connected with aggressive metastasis (17). The conflicting evidence casts doubt on CD133 as a cancer stem cell marker. CD133 has been analyzed via several methods including Western blot analysis, immunomagnetic (MACS), and fluorescence-activated cell sorting (FACS) $(1,18,19)$, however the vast majority of CD133 research is carried out using the AC133 monoclonal antibody, and to a lesser extent the 293C monoclonal antibody $(2,4-6,12)$, both of which detect glycosylated epitopes of CD133. Recent research (20) has shown that these antibodies are not specific for a glycosylated epitope, and that AC133 can detect protein with mutated glycosylation sites, as well as non-glycosylated recombinant CD133 protein. Other researchers (21) have shown that 'pan' antibodies that bind directly to non-glycosylated epitopes of CD133, can be used as viable alternatives to AC133. Importantly, it is becoming clear that there are many CD133 variants $(10,22)$, and that the reagents commonly used may not recognize all of them. 
In this study, we analyzed CD133 expression in immortalized and primary GBM cell lines using antibodies against glycosylated and non-glycosylated epitopes to compare CD133 expression to CD133 glycosylation. Using the abundance of CD133 protein in Caco-2 colon cancer cells as a positive control, we determined that the antibodies specific for glycosylated epitopes did not detect all CD133 protein expressed in GBM cells.

\section{Materials and methods}

Cell culture. Primary GBM cell lines were derived from tumors obtained from consenting patients undergoing surgery at Wellington Hospital. Primary cells and the immortalized glioblastoma cell line LN18 were cultured in RPMI-1640 medium. The immortalized colon cancer cell line, Caco-2, was cultured in Dulbecco's Minimal Essential Medium. All media was supplemented with $10 \%$ fetal bovine serum (FBS; Sigma, $\mathrm{NZ}$ ), $100 \mathrm{U} / \mathrm{ml}$ penicillin, $100 \mu \mathrm{g} / \mathrm{ml}$ streptomycin, and $2 \mathrm{mM}$ glutamax. Cell culture reagents were from Invitrogen (NZ).

RNA extraction and real-time PCR. RNA was extracted (ZR RNA Mini Prep kit, Zymo Research, CA, USA) following the manufacturer's protocol. cDNA was synthesized using I-Script cDNA Synthesis (BioRad, NZ). Real-time PCR was performed using primers to 18s (QT00199367) and CD133 (QT00075586; Qiagen, CA, USA), with QuantiTect SYBR Green PCR Master Mix (Qiagen) in the 7500 real-time PCR System (Applied Biosystems, CA, USA), according to manufacturer's instructions. Cycle threshold $(\mathrm{Ct})$ for $\mathrm{CD} 133$ were normalized to $18 \mathrm{~s}$ rRNA $\mathrm{Ct}(\Delta \mathrm{Ct}) . \Delta \mathrm{Ct}$ were normalized between the vehicle and treated cell RNA $(\Delta \Delta \mathrm{Ct})$ and fold change determined by $2^{-\Delta \Delta C t}$. Amplification efficiency of all primers were equivalent (Qiagen website). The CD133 primers detected all known mRNA variants, binding position between base pairs 800-900 or 1100-1200 depending on transcript. Caco-2 cells were used as a positive control for CD133 expression.

Western blot analysis. Cells were lysed in $140 \mathrm{mM} \mathrm{NaCl}$, $50 \mathrm{mM}$ Tris ( $\mathrm{pH} 7.5-8.0$ ), 1\% Triton X-100, 1X Protease Inhibitor, $0.1 \%$ SDS. Insoluble debris was pelleted, supernatant collected, and 1 vol of loading buffer [1X Tris.Cl/SDS (pH 6.8), 20\% glycerol, 40 mg/ml SDS, $2 \%$ 2-mercaptoethanol, $0.01 \mathrm{mg} / \mathrm{ml}$ bromophenol blue] added. Protein was denatured at $95^{\circ} \mathrm{C}$ for $5 \mathrm{~min}$ before storage at $-20^{\circ} \mathrm{C}$. Protein was quantified using the Bio-Rad Dc Protein Assay (Bio-Rad), according to manufacturer's instructions. Absorbance at $750 \mathrm{~nm}$ was read on a Versamax microplate reader (Molecular Devices, CA, USA). Fifty micrograms of protein was run in $10 \%$ SDS-PAGE and transferred to PVDF membrane (Bio-Rad). Membranes were stained with amido black $[0.1 \%(\mathrm{w} / \mathrm{v})$ amido black $/ 10 \%$ acetic acid] to visualize loading before blocking in $5 \%(\mathrm{w} / \mathrm{v}) \mathrm{BSA}$ for $1 \mathrm{~h}$ at room temperature. Membranes were incubated overnight at $4^{\circ} \mathrm{C}$ in $5 \% \mathrm{BSA}$ with either 1:500 rabbit monoclonal anti-CD133 antibody (C24B9; Cell Signaling Technology, MA, USA) or 1:100 mouse monoclonal anti-CD133 antibody (32AT1672; Abcam, UK). Approximate binding sites are shown in Fig. 1. Secondary antibodies conjugated to peroxidase (goat antirabbit or anti-mouse, Santa Cruz Biotechnology, CA, USA) were used at 1:2000, and then detected with SuperSignal West Pico Chemiluminescent Substrate (Thermoscientific, MA, USA) and autoradiography (X-Omat, Kodak, CA, USA).

Flow cytometry. Cells, suspended in HBSS (Invitrogen) with $2 \%$ FBS (Sigma) at $2 \times 10^{6}$ cells $/ \mathrm{ml}$, were incubated for $1 \mathrm{~h}$ at room temperature with either 1:100 mouse IgG1-PE and mouse IgG2b-APC isotype control antibodies, or 1:100 human CD133/1 AC133-PE and human CD133/2 293C-APC antibodies (Miltenyi Biotech, Germany). Cells were washed, then analyzed on a BD FACSort (Becton-Dickinson, CA, USA). FlowJo 8.8.6 software (TreeStar, CA, USA) was used for data analysis.

Immunofluorescence. Caco-2 and 0906 cells were cultured on chamber slides and fixed with cold $95 \%$ ethanol $/ 5 \%$ acetic acid. Fixed and dried cells were blocked with $2 \%$ FBS (Sigma) in HBSS (Invitrogen) for $1 \mathrm{~h}$ at room temperature. Cells were incubated overnight at $4^{\circ} \mathrm{C}$ with 1:50 32AT1672 (mouse) and 1:100 C24B9 (rabbit). Cells were washed and incubated $1 \mathrm{~h}$ at room temperature in the dark with 1:1000 goat anti-mouse IgG Alexa 488 (Invitrogen) and 1:1000 goat anti-rabbit Alexa 555 (Invitrogen). After washing, Prolong Gold anti-fade reagent with DAPI (Invitrogen) was applied and fluorescence visualized on a FV1000 confocal microscope (Olympus, NZ) with Fluoview FV1000 software (Olympus).

\section{Results}

Expression of CD133 in glioblastoma cells. A panel of primary human glioblastoma cell cultures were analyzed for CD133 expression, using the colon cancer cell line Caco-2 as a positive control. Real-time PCR analysis (Fig. 2a) revealed that each glioblastoma cell line expressed some CD133 mRNA, although at a lower level than the Caco-2 positive control. The exception was the 0713 cell line, which expressed no detectable levels of CD133 mRNA. CD133 transcription was compared to protein expression in the same cell lines by Western blot analysis, using the C24B9 monoclonal antibody that recognizes a non-glycosylated epitope in the second large extracellular loop (Fig. 2b). The protein expression correlated with the transcript levels in most cases, indicating a protein band in the Caco- 2 cells and most of the GBM cells. The 0713 cells had no protein band, which correlated with the absence of a CD133 transcript. Interestingly, the 0810 and 0809 cell lines did not show a detectable protein band despite the presence of a detectable transcript, suggesting a control point between CD133 transcription and protein translation. Surprisingly, all of the glioblastoma cell lines where CD133 protein was detected had bands that were approximately $16 \mathrm{kDa}$, significantly smaller than the molecular weight of the full-length CD133 expressed in Caco-2 cells, approximately $115 \mathrm{kDa}$. A second CD133 antibody, 32AT1672 monoclonal antibody, that binds an epitope nearer the $\mathrm{NH}_{2}$ terminus of the protein (Fig. 1) was also used in Western blot analysis. This did not detect any CD133 in the glioblastoma cell lines (Fig. 2c), while the Caco-2 positive control had a detectable protein band at the expected molecular weight, suggesting the variant had lost some of the $\mathrm{NH}_{2}$ portion of the full-length molecule. Flow cytometric analysis was used to measure 


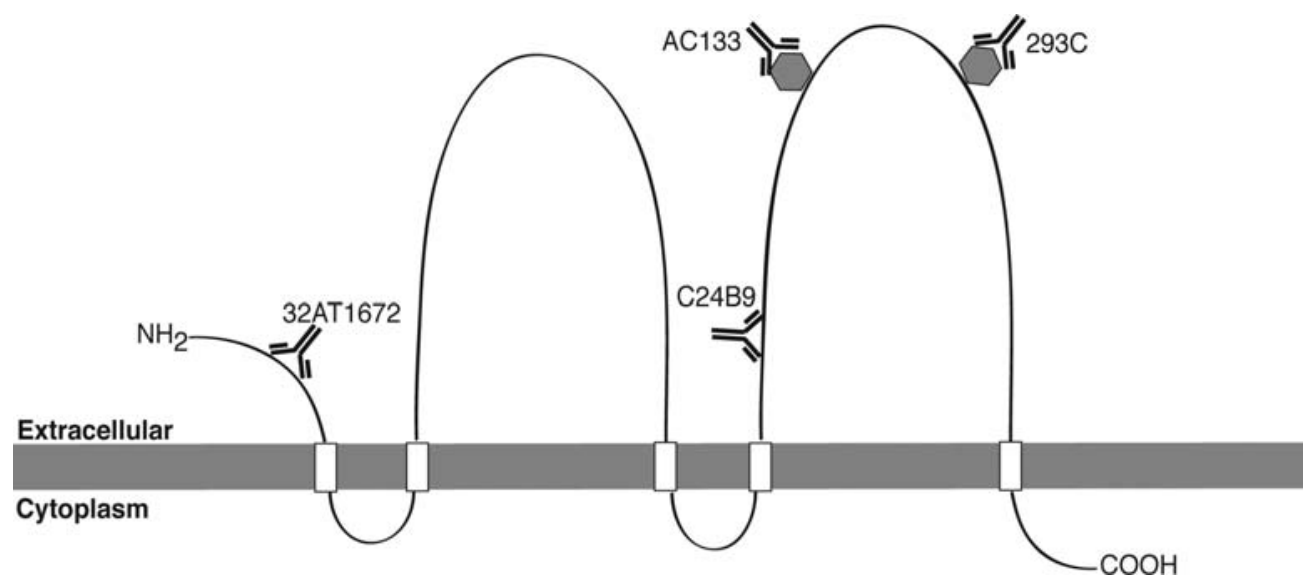

Figure 1. Diagram of CD133 with approximate locations of monoclonal pan-antibodies C24B9 and 32AT1672, and glycosylated epitopes for monoclonal antibodies AC133 and 293C.

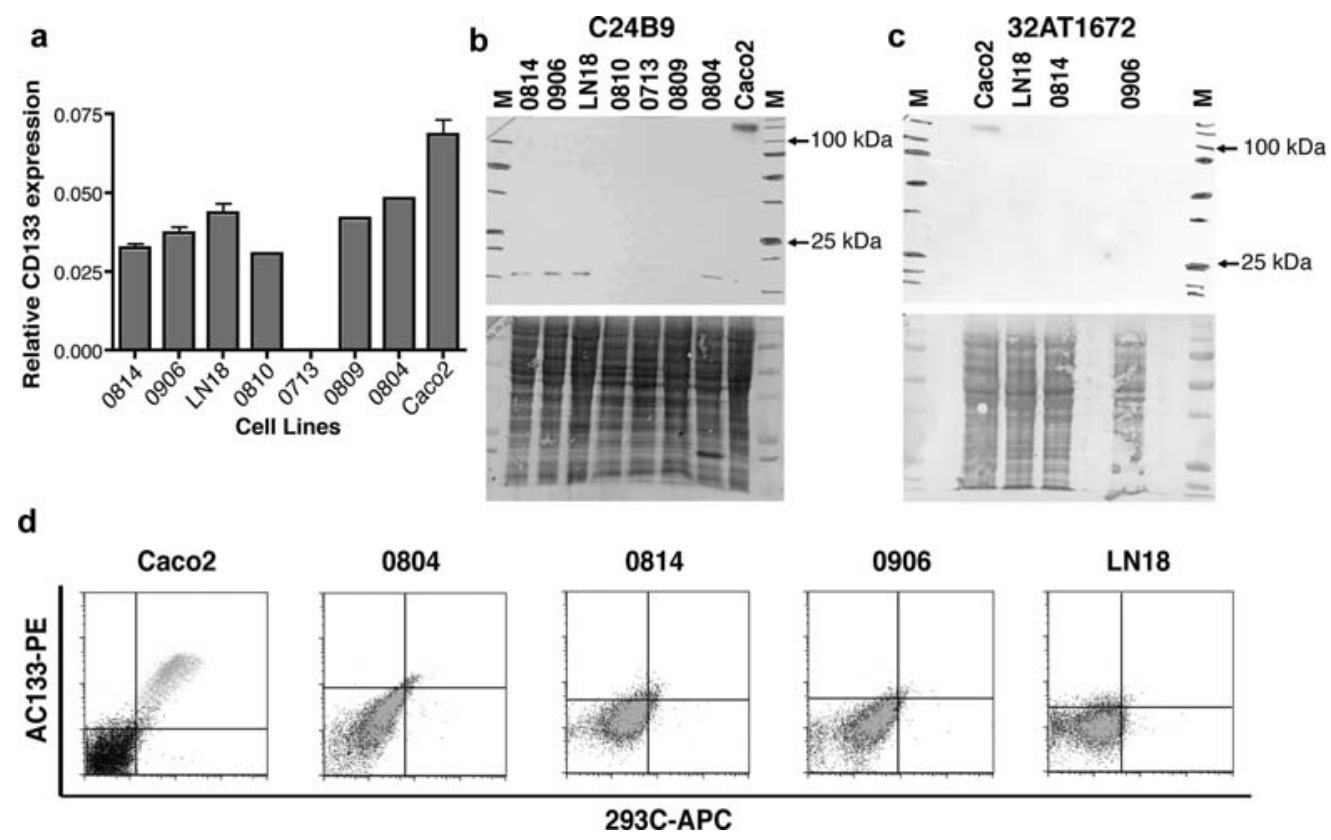

Figure 2. Expression of CD133 mRNA (a), protein (b and c), and protein glycosylation (d) in glioblastoma cells. (a) Real-time PCR analysis of CD133 mRNA in the Caco-2 cell line and a panel of glioblastoma cell lines, $\Delta$ Ct normalized to 18 s ribosomal RNA. (b and c) Representative Western blot analysis of CD133 protein in the Caco-2 cell line and a panel of glioblastoma cell lines, using antibodies C24B9 (b) and 32AT1672 (c). M indicates control lanes with known molecular weight protein bands. Amido black staining of the membrane was used as a control for input protein loading. (d) Flow cytometric analysis of CD133 protein glycosylation in the Caco-2 and glioblastoma cell lines. The 293C and AC133 antibodies (grey) were compared to matched isotype controls (black). Shown are representatives of three replicate experiments.

glycosylation and surface expression of CD133. Both the AC133 and 293C antibodies, which recognize glycosylated epitopes of CD133, stained very few GBM cells at a very low level. In contrast, Caco-2 cells exhibited strong expression of glycosylated CD133 at the cell surface (Fig. 2d).

Location of CD133 protein in 0906 and Caco-2 cells. Lack of recognition by $\mathrm{AC} 133$ and $293 \mathrm{C}$ could indicate loss of either glycosylation, or of surface localization of CD133. The cellular location of the full-length and short CD133 protein was determined by immunofluorescence and confocal microscopy (Fig. 3a). In Caco-2 cells, the C24B9 antibody showed that CD133 protein was present predominantly in the membrane of the cells (Fig. 3b). The 32AT1672 epitope was detected at a much lower level, correlating with the lower intensity of the protein band shown in Fig. 2c, and showed distribution throughout the membrane, and in small clusters in the cytoplasm. In comparison, GBM 0906 cells did not show membrane localization of CD133. The C24B9 antibody showed distribution of the protein throughout the cytoplasm, at a lower concentration than in the Caco-2 cells. The 32AT1672 antibody was not detected at all in the GBM line, correlating with the Western blot analysis (Fig. 2c).

Stress-induced expression of CD133. CD133 expression has been reported to change in response to stresses such as hypoxia 
a
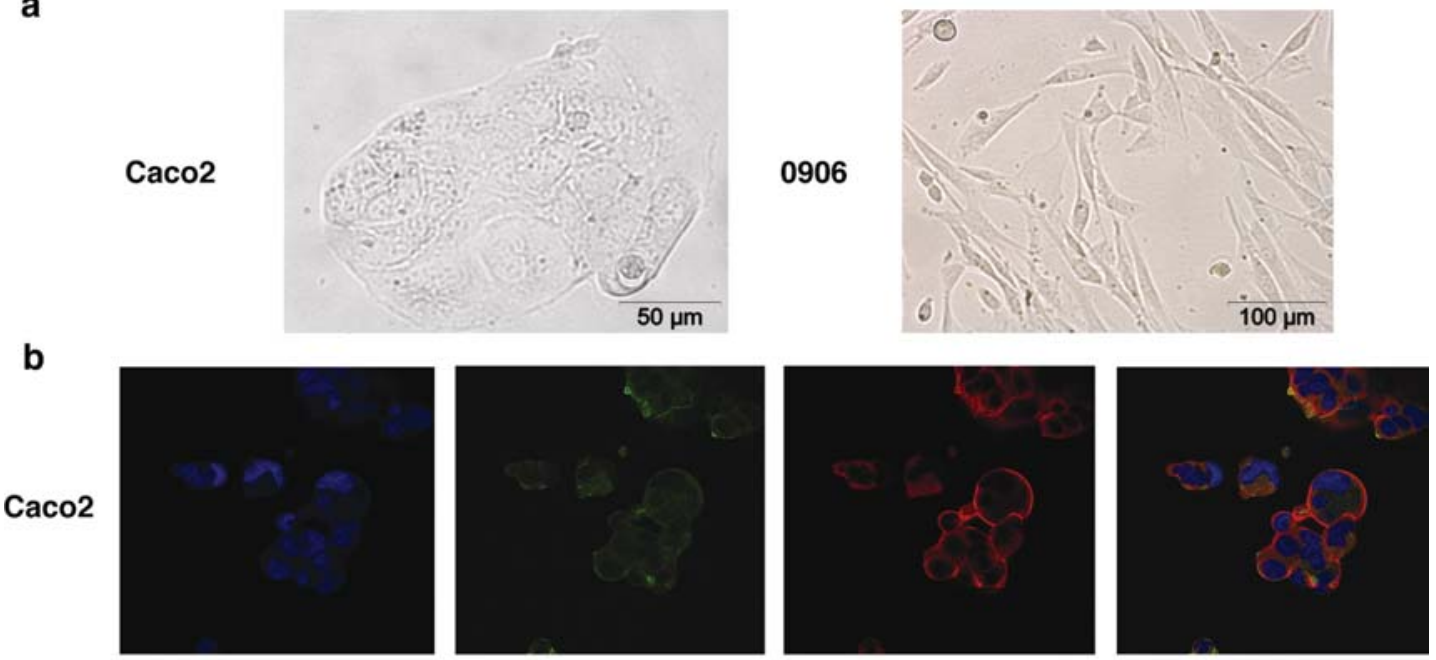

0906

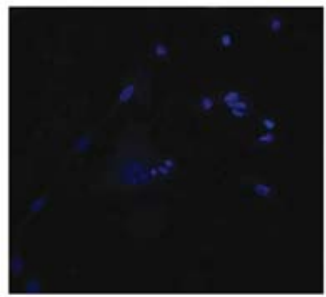

DAPI

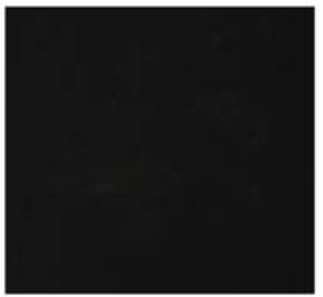

32AT1672

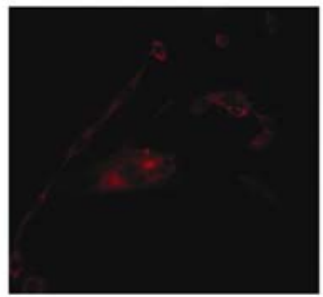

C24B9

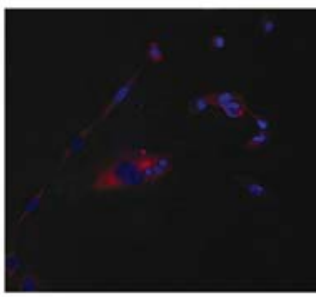

Merge

Figure 3. Light (a) and confocal (b) microscopy of Caco-2 and 0906 cells. (a) Bright field images of Caco-2 (x40) and 0906 (x20) cells. (b) Immunofluorescence of CD133 in Caco-2 (x40) and 0906 (x20) cells, using the 32AT1672 (green) and C24B9 (red) antibodies. Nuclei are stained with DAPI (blue). The merge includes all three frames.
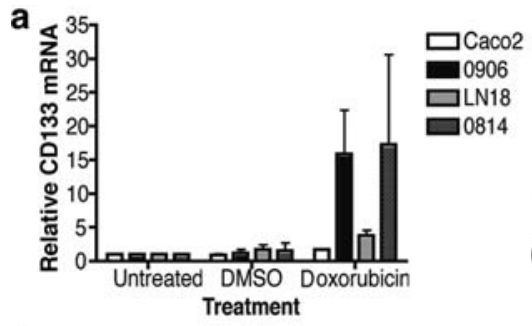

b

C

Caco2
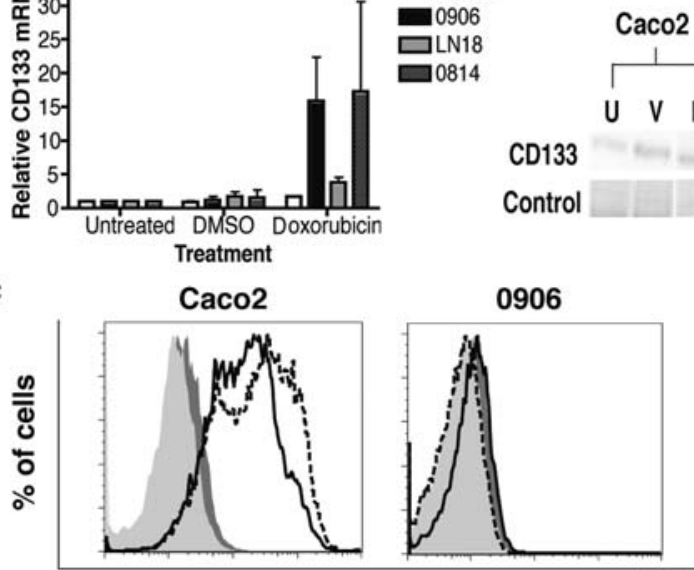

CD133

(kat $\leftarrow 115 \mathrm{kDa}$

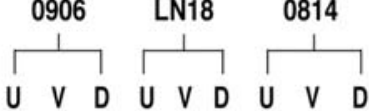

Control
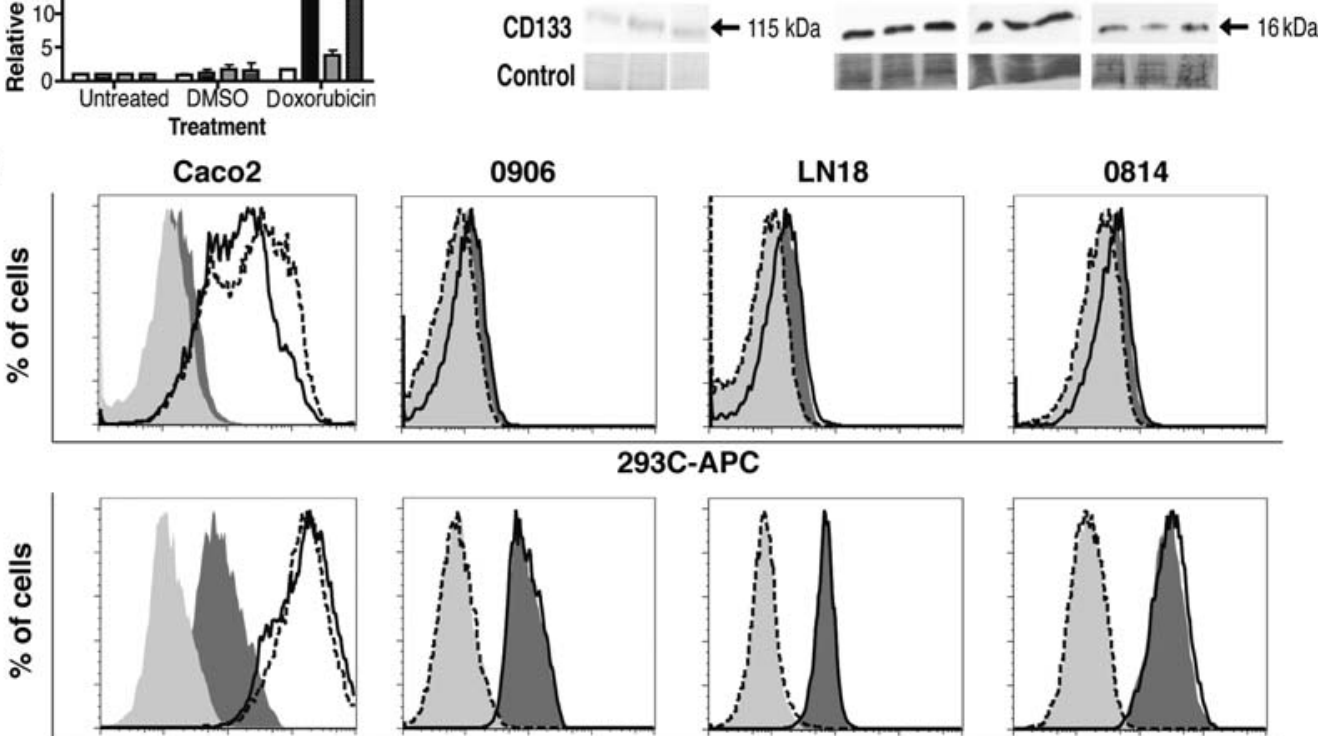

293C-APC

\section{AC133-PE}

Figure 4. Stress-induced expression of CD133 mRNA (a), protein (b), and protein glycosylation (c) in glioblastoma cells. (a) Real-time PCR analysis of CD133 mRNA in the Caco-2 and glioblastoma cell lines, with $\Delta \mathrm{Ct}$ normalized to $18 \mathrm{~s}$ ribosomal RNA. Treated samples were normalized to untreated samples. (b) Representative Western blot analysis of CD133 protein in Caco-2 and glioblastoma cell lines, using the C24B9 antibody, comparing untreated (U), DMSO vehicle (V), and $5 \mu \mathrm{M}$ doxorubicin-treated (D). Protein molecular weight sizes are indicated. Amido black staining of the membrane was used as a control for input protein loading. (c) Flow cytometric analysis of CD133 protein glycosylation in Caco-2 and glioblastoma cell lines. Doxorubicin-treated samples (black line), with isotype control (dark grey fill), were compared to DMSO vehicle control samples (black dashed line), with isotype control (light grey fill). Shown are representatives of three replicate experiments. 
(19) and chemotoxic stress (23). In the absence of a known function for CD133, we used this stress response to examine the potential functionality of the short variant. Caco-2 cells and a subset of the immortalized and primary glioblastoma cell lines were stressed by exposure to $5 \mu \mathrm{M}$ of the chemotoxic agent doxorubicin for $24 \mathrm{~h}$. Expression of CD133 mRNA and protein in stressed cells was compared to untreated and vehicle (DMSO)-treated cells. Real-time PCR analysis indicated a marked increase in CD133 mRNA in the 0906 and 0814 stressed cell lines, indicating a positive correlation between chemotoxic stress and CD133 transcription in glioblastoma cells (Fig. 4a). This finding correlated with a minor increase in CD133 protein expression detected by Western blot analysis (Fig. 4b). The Caco-2 cells, and the immortalized GBM cell line LN18, showed no detectable changes in protein band intensity, correlating with the minimal change in mRNA expression. The effect of stress on CD133 protein glycosylation was analyzed by flow cytometry and showed no significant change in any cell line in response to stress (Fig. 4c). While this correlates with the absence of a change in CD133 mRNA and protein expression in Caco- 2 and LN18 cells, the absence of a change in protein glycosylation in the 0906 and 0814 cells did not correlate with the increased CD133 mRNA and protein levels. This suggests the increased protein expression in the primary GBM lines was not reaching the surface, or was not glycosylated, consistent with the data in Fig. 3.

\section{Discussion}

CD133 protein has previously been described $(5,9)$ as an abundant protein in the membrane of colon cancer cells, as well as normal colon cells. This was consistent with our data demonstrating high levels of CD133 mRNA and protein in Caco- 2 cells. The protein was detected at high levels by using both the 'pan' C24B9 antibody in a Western blot analysis, and using the AC133 and 293C antibodies specific for glycosylated epitopes in flow cytometric analysis. Previous research (23) has also described AC133+ CD133 cells occurring frequently in GBM. In contrast we found no detectable glycosylation and low levels of protein in our primary GBM cells compared to the Caco- 2 cells. However, in the primary GBM cells the mRNA levels were substantial, indicating that low protein level may be due to a possible translational and/or posttranslational control. Culture conditions, including exposure to serum, have been hypothesized to affect CD133 expression or glycosylation (19). This could potentially explain why our primary GBM lines had less CD133 glycosylation-positive cells than others have reported. While there was little or no glycosylation detected, CD133 was detected in most cells by microscopy. This indicated that the AC133 and 293C epitopes were not effective in analyzing overall CD133 protein.

Our GBM cells would have been termed CD133-negative using the AC133 and 293C antibodies, despite the definitive CD133 band detected using the C24B9 antibody. However, the CD133 band was $\sim 16 \mathrm{kDa}$, significantly smaller than the full-length protein in Caco-2 cells. This short CD133 protein could be just a degradation product, or it may be a specific cleavage product or splice variant that has some effect on the processes of the cell. The 32AT1672 antibody did not detect a protein in any GBM cells, suggesting that degradation of
CD133 was less likely, as the N-terminus should be represented in any non-specific degradation product. Instead, the short CD133 variant seen with the C24B9 antibody may be a specific splice variant or cleavage product, where the 32AT1672 epitope has been cleaved. The C24B9 antibody binds to the second extracellular loop (Fig. 1), so therefore the short variant protein is a $16 \mathrm{kDa}$ section of the second loop and the surrounding area, possibly part of the transmembrane domain. However, the protein sequence would need to be determined to confirm this hypothesis.

Full-length CD133 protein is located in the plasma membrane of cells (1). This was consistent with our confocal microscopy analysis on Caco- 2 cells, showing the location of the protein by immunofluorescence. In GBM cells, CD133 did not localize to the membrane, but instead was found throughout the cytoplasm. This explained why there was no glycosylation detected by flow cytometry, as the protein was not on the cell surface. Further, the short variant may not contain the necessary sites for glycosylation. The only increase seen in glycosylation due to stress was a small increase in AC133 mean fluorescence intensity (MFI) in the GBM cell lines. However, further analysis showed that doxorubicin caused cell cycle arrest in G2 phase (data not shown) and previous data have demonstrated that cells in G2 phase have a higher level of AC133 glycosylation (6), which would account for the very small increase in glycosylation observed.

The CD133 protein does not have a known function $(4,5)$, making it difficult to determine the function of the short variant. Previous research $(19,23)$ has shown a change in CD133 protein expression due to different stressors, including hypoxia and chemotoxic stress, indicating a possible relationship between stress and CD133 production and function. Therefore, we used chemotoxic stress to determine a possible function of the small variant. We discovered a large increase in CD133 mRNA, indicating that CD133 transcription was up-regulated due to stress. While there was a large increase in mRNA, there was only a minor increase in protein, again suggesting a control point between transcription and translation preventing the full expression of the protein. However, the increased expression in response to stress supported our hypothesis that the small variant is a specific cleavage product or splice variant, rather than a non-specific degradation product.

Previous research $(3,4,6,17)$ has indicated opposing views of the CD133 protein in relation to stem and stem-like cells. Data are inconsistent as to whether CD133-negative cells have stem-like properties. The method commonly used to designate cells as CD133-negative and -positive uses the antibodies that recognize the glycosylated epitopes AC133 and 293C. Our research has shown that in GBM cells, AC133-negative cells express CD133, albeit a small variant. Similar analysis using AC133-negative melanoma xenograft cells indicated a band of $\sim 16 \mathrm{kDa}$ using the C24B9 antibody in Western blot analysis, while AC133-positive cells had the full-length band (unpublished observation, McConnell).

In summary, AC133-negative cells may in fact be CD133positive, as they contain a small CD133 variant that may be functional. This reinforces recent discussion $(20,21)$ that CD133 analysis should not focus on specific glycosylated epitopes, but should encompass all CD133 variants. The inclusion of alternate $\mathrm{CD} 133$ protein products may produce 
more consistent results for the analysis of CD133 biology in stem cell, and cancer stem cell, research.

\section{Acknowledgements}

This project was funded by the Sir Roy McKenzie Medical Research Fellowship awarded to Dr M.J. McConnell.

\section{References}

1. Weigmann A, Corbeil D, Hellwig A and Huttner WB: Prominin, a novel microvilli-specific polytopic membrane protein of the apical surface of epithelial cells, is targeted to plasmalemmal protrusions of non-epithelial cells. Proc Natl Acad Sci USA 94: 12425-12430, 1997.

2. Yin AH, Miraglia S, Zanjani ED, et al: AC133, a novel marker for human hematopoietic stem and progenitor cells. Blood 90: 5002-5012, 1997.

3. Pallini R, Ricci-Vitiani L, Banna GL, et al: Cancer stem cell analysis and clinical outcome in patients with glioblastoma multiforme. Clin Cancer Res 14: 8205-8212, 2008.

4. Dell'Albani P: Stem cell markers in gliomas. Neurochem Res 33: 2407-2415, 2008.

5. LaBarge MA and Bissell MJ: Is CD133 a marker of metastatic colon cancer stem cells? J Clin Invest 118: 2021-2024, 2008.

6. Jaksch M, Munera J, Bajpai R, Terskikh A and Oshima RG: Cell cycle-dependent variation of a CD133 epitope in human embryonic stem cell, colon cancer, and melanoma cell lines. Cancer Res 68: 7882-7886, 2008.

7. Chang CJ, Hsu CC, Yung MC, et al: Enhanced radiosensitivity and radiation-induced apoptosis in glioma CD133-positive cells by knockdown of SirT1 expression. Biochem Biophys Res Commun 380: 236-242, 2009.

8. Baba T, Convery PA, Matsumura N, et al: Epigenetic regulation of CD133 and tumorigenicity of CD133+ ovarian cancer cells Oncogene 28: 209-218, 2009.

9. Neuzil J, Stantic M, Zobalova R, et al: Tumour-initiating cells vs. cancer 'stem' cells and CD133: What's in the name? Biochem Biophys Res Commun 355: 855-859, 2007.
10. Miraglia S, Godfrey W, Yin AH, et al: A novel five-transmembrane hematopoietic stem cell antigen: Isolation, characterization, and molecular cloning. Blood 90: 5013-5021, 1997.

11. Yi JM, Tsai HC, Glockner SC, et al: Abnormal DNA methylation of CD133 in colorectal and glioblastoma tumors. Cancer Res 68: 8094-8103, 2008.

12. Shmelkov SV, Butler JM, Hooper AT, et al: CD133 expression is not restricted to stem cells, and both CD133(+) and CD133(-) metastatic colon cancer cells initiate tumors. J Clin Invest 118: 2111-2120, 2008.

13. Nicolis SK: Cancer stem cells and 'stemness' genes in neurooncology. Neurobiol Dis 25: 217-229, 2007.

14. Hide T, Takezaki T, Nakamura H, Kuratsu J and Kondo T: Brain tumor stem cells as research and treatment targets. Brain Tumor Pathol 25: 67-72, 2008.

15. Fomchenko EI and Holland EC: Stem cells and brain cancer. Exp Cell Res 306: 323-329, 2005.

16. Vezzoni L and Parmiani G: Limitations of the cancer stem cell theory. Cytotechnology 58: 3-9, 2008.

17. Joo KM, Kim SY, Jin X, et al: Clinical and biological implications of CD133-positive and CD133-negative cells in glioblastomas. Lab Invest 88: 808-815, 2008.

18. Jin F, Zhao L, Zhao HY, et al: Comparison between cells and cancer stem-like cells isolated from glioblastoma and astrocytoma on resistance-associated protein genes. Neuroscience 154: 541-550, 2008.

19. Platet N, Liu SY, El Atifi M, et al: Influence of oxygen tension on CD133 phenotype in human glioma cell cultures. Cancer Lett 258: 286-290, 2007.

20. Kemper K, Sprick MR, de Bree M, et al: The AC133 epitope, but not the CD133 protein, is lost upon cancer stem cell differentiation. Cancer Res 70: 719-729, 2010.

21. Bidlingmaier S, Zhu X and Liu B: The utility and limitations of glycosylated human CD133 epitopes in defining cancer stem cells. J Mol Med 86: 1025-1032, 2008.

22. Corbeil D, Joester A, Fargeas CA, et al: Expression of distinct splice variants of the stem cell marker prominin-1 (CD133) in glial cells. Glia 57: 860-874, 2009.

23. Liu G, Yuan X, Zeng Z, et al: Analysis of gene expression and chemoresistance of CD133+ cancer stem cells in glioblastoma. Mol Cancer 5: 67, 2006. 